\section{Superconductivity enhanced by Hg fission}

Eor the successful application of high- temperature copper oxide superconductors, the problem of the ease of motion of magnetic vortices (quantized flux lines) within the material must be solved. The motion results in finite electrical resistance which prevents the desired loss-free conduction of current ${ }^{1}$. We demonstrate a solution to this problem by anchoring the vortices with crystallographic defects induced by fission of mercury atoms in a mercury/copper oxide superconductor.

Easy vortex motion, the origin of which is intrinsic to the material, can be counteracted by correlated disorder in the form of columnar defects ${ }^{2}$. Such defects effectively

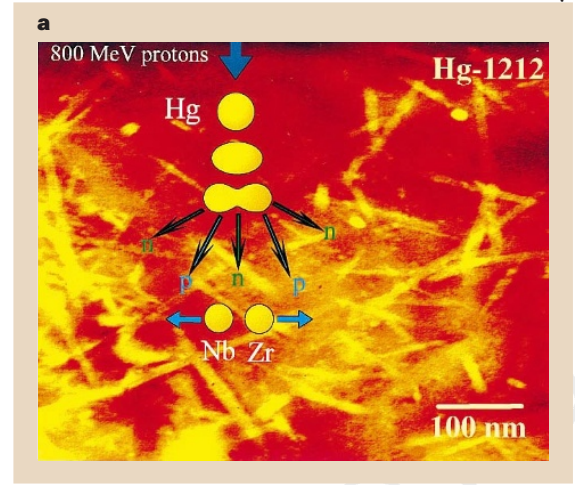

localize vortices ${ }^{1}$ and expand the range of finite critical current density $J_{c}$ (marked by the irreversibility line $e^{1}$ in the field-temperature $(H-T)$ diagram $)^{2,3}$. One possibly technologically relevant way to produce an effective columnar defect structure is by irradiation with energetic $(\sim 0.2-0.8 \mathrm{GeV})$ protons, which will induce nuclear fission of sufficiently heavy nuclei ${ }^{4}$. The resulting fission fragments could in turn produce columnar damage tracks ${ }^{5}$, as has been shown for ${ }_{83}^{209} \mathrm{Bi}$ nuclei ${ }^{6}$. There is no directionality in the fission process, so the resulting tracks will be highly misaligned (splayed $)^{6}$. The action of splay is to force the entanglement of vortices ${ }^{7}$, expected to reduce thermal activation (creep) of vortices out of columnar tracks and to enhance $J_{c}$ even further.

In any superconductor, the irreversibility line in the $H-T$ space, above which the vortex matter 'liquefies' and is very mobile ${ }^{1}$, is ultimately limited by the transition temperature $T_{c}$. So the question arising is whether the fission process can be realized in mercury/copper oxides - materials with the highest $T_{\mathrm{c}}$ found to date ${ }^{8,9}$. The answer will depend on three factors: the experimentally unknown fission cross-section $\sigma$
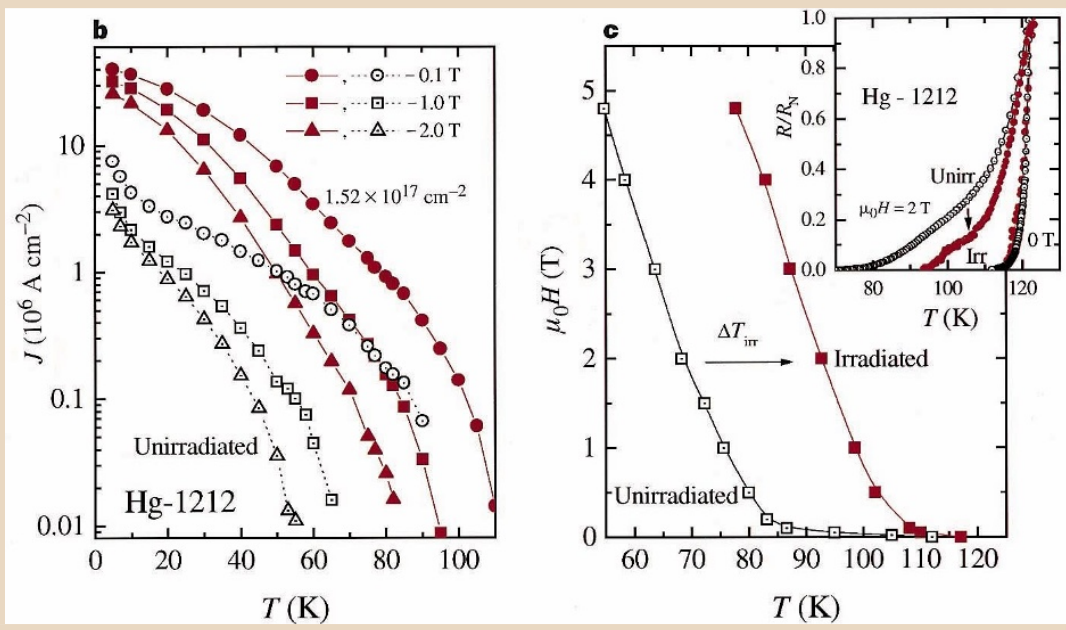

Figure 1 a, Transmission electron micrograph of $\mathrm{Hg}-1212$ bulk superconductor prepared as in ref. 9, irradiated with 0.8-GeV protons. Fission track diameter, $~ 80-90 \AA \AA$; defect density, $\sim 5.9 \times 10^{10} \mathrm{~cm}^{-2} ; \sigma \approx 110 \mathrm{mbarns} ; \mathrm{Hg}$ density, $\sim 5.3 \times 10^{21} \mathrm{~cm}^{-3}$; density of events, $8.9 \times 10^{13}$ fissions $\mathrm{cm}^{-3}$. Mean track length implied is $\sim 6.6 \mu \mathrm{m}$, consistent with calculations $s^{5,6}$. Sketch illustrates the fission process. On entering a mercury nucleus, a $0.8-\mathrm{GeV}$ proton collides with nucleons. The nucleus 'boils off' protons and neutrons, which carry off about $0.6 \mathrm{GeV}$ energy. The remaining energy is split between the fission daughters. $\mathbf{b}$, Persistent current density $J$ against temperature for $\mathrm{Hg}-1212$ before and after proton exposure, for three applied magnetic fields. Bulk $J$ is above $10^{7} \mathrm{~A} \mathrm{~cm}^{-2}$. After irradiation $J$ becomes large above $100 \mathrm{~K}$, well above the irreversibility line of unirradiated sample. c, Irreversibility lines of unirradiated and 0.8-GeV proton-irradiated Hg-1212 obtained from the onset of nonlinear current-voltage characteristics. Arrow, $25 \mathrm{~K}$ expansion at $\mu_{0} \mathrm{H}=2 \mathrm{~T}$ on irradiation. Inset, resistance $R$ (normalized to $R$ at $T_{\mathrm{c}}$ ) before and after irradiation. In finite fields onset of linear resistance (dissipation) is shifted to higher temperatures and normalized $R$ at any $T$ is significantly reduced (arrow) by proton irradiation. for a ${ }_{80}^{200} \mathrm{Hg}$ fission process launched with fast protons, the as-yet unknown threshold of electronic energy loss rate for columnar track formation ${ }^{5}$ in mercury/copper oxides by the fission fragments, and the efficacy of splayed tracks in these cuprates.

We exposed polycrystalline samples of $\mathrm{HgBa}_{2} \mathrm{CaCu}_{2} \mathrm{O}_{6+\delta}(\mathrm{Hg}-1212)^{9}$ to $0.8-\mathrm{GeV}$ protons (fluence of $1.52 \times 10^{17} \mathrm{~cm}^{-2}$ ) at Los Alamos National Laboratory (backed by the Electric Power Research Institute and NSF) using the proton beam from Los Alamos Meson Physics facility at the Weapons Neutron Research branch. The result was the first experimental record of a mercury fission process by which we could install splayed tracks in a Hg-1212 superconductor with transition temperature $T_{c}$ of roughly $120 \mathrm{~K}$. A cross-sectional transmission electron microscope image of Hg-1212 (Fig. 1a) illustrates the splayed columnar nature of the damage, typical of a fission process.

The effect on current conduction is shown in Fig. 1b, c. Before irradiation the persistent current density $J$ in high magnetic fields falls off rapidly with temperature - by three orders of magnitude at $\mu_{0} H=2$ tesla and $T \approx 60 \mathrm{~K}$. After irradiation, $J$ is enhanced by orders of magnitude in fields of several tesla and the regime of finite $J$ is extended to $T>100 \mathrm{~K}$ (higher than in yttrium-, bismuth-, or thallium-based materials $\left.s^{2,3}\right)$. Even for the relatively low proton fluence used here, the large shift in the irreversibility temperature $\Delta T_{\text {irr }} \approx 25 \mathrm{~K}$ is maintained in a field of up to 5.5 tesla (Fig. 1c).

The large shift in $T_{\text {irr }}$ is possibly a result of the renormalization of splay angles ${ }^{1}$ by sizeable superconductive anisotropy of $\mathrm{Hg}$ 1212 (refs 8,9). A uniform splay distribution will not work in a less anisotropic material, such as yttrium-barium-copper oxides, where for splay angles greater than $10^{\circ}$ the motion of vortices is enhanced ${ }^{10}$. Our results imply that the fission process can be extended to higher proton fluences and to mercury/copper oxides with $T_{c}$ above $130 \mathrm{~K}$, for which a wire and tape fabrication process has recently been developed ${ }^{11}$.

L. Krusin-Elbaum, D. Lopez

IBM T. J. Watson Research Center,

Yorktown Heights, New York 10598, USA

\section{J. R. Thompson}

Department of Physics, University of Tennessee,

Knoxville, Tennessee 37996, USA

and Oak Ridge National Laboratory,

Oak Ridge, Tennessee 37831, USA

R. Wheeler

The Ohio State University,

Columbus, Ohio 43210, USA

J. Ullmann

Los Alamos National Laboratory,

Los Alamos, New Mexico 87545, USA

C. W. Chu, Q. M. Lin

Texas Center for Superconductivity,

Houston University, Houston, Texas 77204, USA 
1. Blatter G. etal. Rev. Mod. Phys. 66, 1125 (1994).

2. Civale, L. et al. Phys. Rev. Lett. 67, 648-651 (1991).

3. Budhani, R. C., Suenaga, M. \& Liou, S. H. Phys. Rev. Lett. 69, 3816-3819 (1992).

4. Becchetti, F. D. et al. Phys. Rev. C 28, 276-279 (1983).

5. Toulemonde, M., Bouffard, S. \& Studer, F. Nucl. Instr. Meth. B 91, 108-123 (1994)

6. Krusin-Elbaum, L. et al. Appl. Phys. Lett. 64, 3331-3333 (1994).

7. Hwa, T., LeDoussal, P., Nelson, D. R. \& Vinokur, V. M. Phys. Rev. Lett. 71, 3545-3548 (1996).

8. Schilling, A., Cantoni, M., Guo, J. D. \& Ott, H. R. Nature 363, 56-58 (1993).

9. Xiong, Q. et al. J. Appl. Phys. 76, 7127-7129 (1994).

10. Krusin-Elbaum, L. et al. Phys. Rev. Lett. 76, 2563-2566 (1996).

11. Meng, R. L. et al. Physica C (in the press).

\section{Glycerol generates}

\section{turgor in rice blast}

Many plant pathogenic fungi are able to penetrate the cuticles of their host plants by elaborating specialized cells known as appressoria $^{1,2}$. The morphology and development of appressoria have been well studied $^{2}$, but little is known about how these cells are able to breach the tough plant surface. We have now found that the appressoria of rice blast fungus (Magnaporthe grisea) use glycerol to generate pressure which ruptures plant cuticles.

Rice blast is the most serious disease of cultivated rice, the staple food for onethird of the world's population ${ }^{3,4}$. The fungus forms heavily melanin-pigmented appressoria that generate enormous turgor. The pressure is applied as a physical force to break the rice leaf cuticle $e^{5,6}$. Turgor can be as great as $8.0 \mathrm{MPa}$ (modal value, 6.0 $\mathrm{MPa})^{5}$, equivalent to 40 times the pressure in a car tyre and is, to our knowledge, the highest recorded in any living organism ${ }^{5}$.

We extracted the contents of appressoria and biochemically characterized them, searching for a metabolically compatible solute responsible for generating the hydrostatic pressure. We grew spores of $M$. grisea in water drops on hydrophobic plastic membranes and allowed them to form appressoria. These generated full turgor over a period of 24-48 h. Gas-liquid chromatography showed that the most abundant solute in appressoria is glycerol.

Glycerol is generated rapidly during germination and germ-tube elongation (Fig. 1a). Here it probably contributes to plasma membrane biosynthesis during initial fungal growth ${ }^{7}$. Glycerol levels decrease at the onset of appressorium formation but rise sharply during turgor generation. This coincides with the collapse of the conidium and germ tube, and concentration of the cytoplasm within the unicellular appressorium (Fig. 1b). Intracellular glycerol concentration is considerably higher at this time $(48 \mathrm{~h})$, being contained in a small volume.

We estimate the mean internal volume of an appressorium as $64 \mu \mathrm{m}^{3}$ assuming that the cell is hemispherical with an internal radius of $3.1 \mu \mathrm{m}(n=100$, s.d. $=0.5)$. The mean concentration of glycerol in an appressorium rose from $0.50 \mathrm{M}$ at $24 \mathrm{~h}$ of development to $3.22 \pm 0.40 \mathrm{M}$ after $48 \mathrm{~h}$. This is a conservative estimate because not all of the cell volume is available for glycerol accumulation. The osmotic potential generated by this concentration of glycerol would be $-8.7 \mathrm{MPa}$ at $20{ }^{\circ} \mathrm{C}$ (assuming that glycerol is an ideal solute).

Glycerol solutions deviate from ideal at high concentrations. The maximum concentration of glycerol for which osmotic potential can be assayed psychro- metrically $^{8}$ is $2.0 \mathrm{M}$, but by extrapolation we estimate that $3.22 \mathrm{M}$ glycerol would generate an osmotic potential of -5.8 $\mathrm{MPa}$. This represents a minimum osmotic potential because living appressoria contain other solutes. The appressorium forms in a drop of water, so the turgor produced is at least $5.8 \mathrm{MPa}$.

To validate these estimates we incubated appressoria in a series of glycerol solutions of varying molarity and measured the frequency of cytorrhysis ${ }^{5}$ (cell collapse). The frequency was dependent on external glycerol concentration. A concentration of $1.75 \mathrm{M}$ glycerol $(-3.7 \mathrm{MPa})$ caused the collapse of $52 \%$ of appressoria (data not

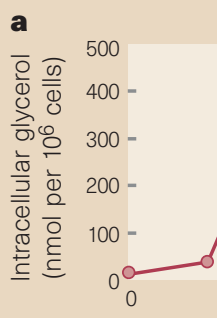

b

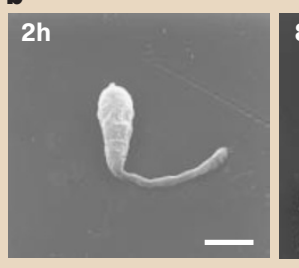

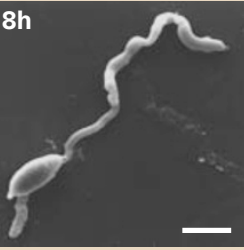

20 Time (h)

30

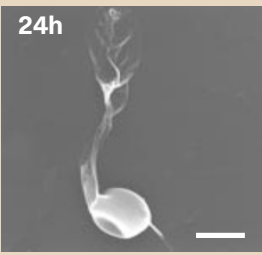

50

Figure 1 Intracellular glycerol increases during M. grisea appressorium turgor generation. a, Change in glycerol contents of extracts made from germinating conidia and developing appressoria with time, determined enzymatically using a glycerol-specific assay (Boehringer). b, Low-temperature scanning electron microscope images of developing appressoria at corresponding times. Appressoria were allowed to form in water drops on polytetrafluoroethylene (PTFE-Teflon, DuPont) membranes. As the appressorium develops turgor the conidium and germ tube collapse ${ }^{6}$. Cytoplasm is present only in the unicellular appressorium. Scale bars, $20 \mu \mathrm{m}$.

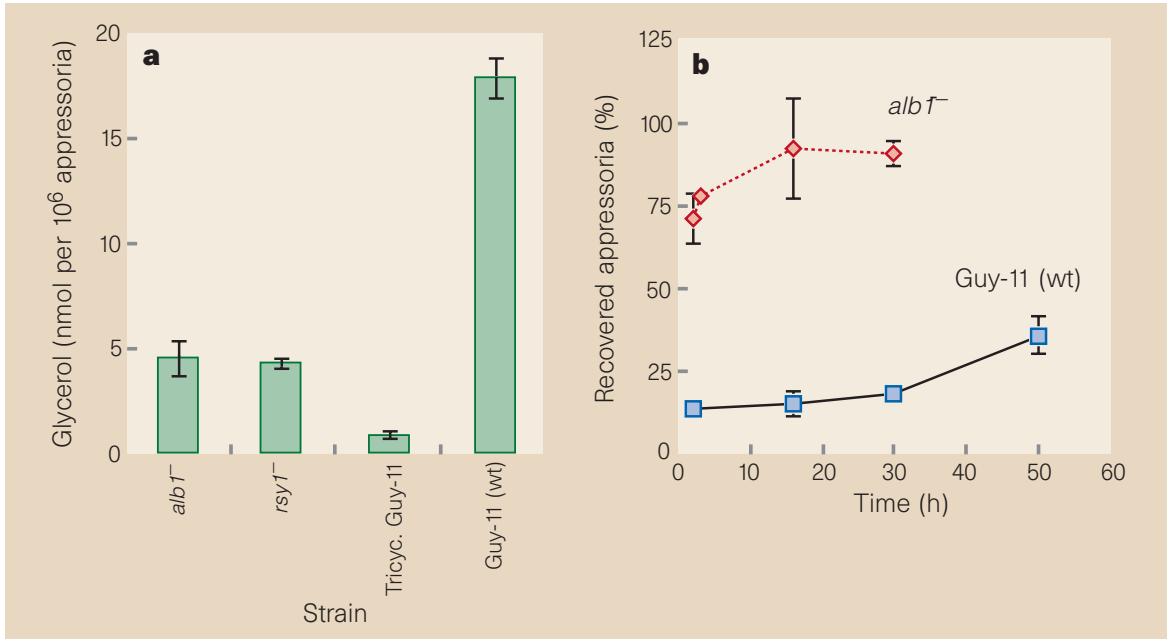

Figure 2 Non-melanized appressoria are permeable to glycerol. a, Intracellular glycerol levels in appressoria of alb1- and rsy 1- mutants $^{9}$; and of strain Guy-11 treated with tricyclazole, compared with wild-type untreated appressoria. We measured intracellular glycerol concentration after $24 \mathrm{~h}$. b, We allowed appressoria to form in water for $24 \mathrm{~h}$ on PTFE membranes and then replaced the water with $4 \mathrm{M}$ aqueous glycerol. We determined the rate of cytorrhysis and the proportion of appressoria that had recovered from cytorrhysis at each time point. Mean ( \pm s.d.) values from 100 appressoria in two independent experiments are represented. Melanized and non-melanized appressoria recovered fully after 60 min incubation in water (not shown). 